\title{
Relaxation and derelaxation of pure and hydrogenated amorphous silicon during thermal annealing experiments
}

\author{
F. Kail, ${ }^{1}$ J. Farjas, ${ }^{1}$ P. Roura,,${ }^{1, a)}$ C. Secouard, ${ }^{2}$ O. Nos, ${ }^{3}$ J. Bertomeu, ${ }^{3}$ F. Alzina, ${ }^{4}$ and \\ P. Roca i Cabarrocas ${ }^{5}$ \\ ${ }^{1}$ Department of Physics, GRMT, University of Girona, Montilivi Campus, E17071 Girona, Catalonia, Spain \\ ${ }^{2}$ LTS, CEA Grenoble, 17 rue des Martyrs, 38054 Grenoble Cedex, France \\ ${ }^{3}$ Dept. Fisica Aplicada and Optica, University of Barcelona, E-08028 Barcelona, Spain \\ ${ }^{4}$ Institut Català de Nanotecnologia (ICN), Campus de la UAB, 08193 Bellaterra, Spain \\ ${ }^{5}$ LPICM, Ecole Polytechnique, 91128 Palaiseau, France
}

(Received 21 May 2010; accepted 24 June 2010; published online 23 July 2010)

\begin{abstract}
The structural relaxation of pure amorphous silicon (a-Si) and hydrogenated amorphous silicon (a-Si:H) materials, that occurs during thermal annealing experiments, has been analyzed by Raman spectroscopy and differential scanning calorimetry. Unlike a-Si, the heat evolved from a-Si:H cannot be explained by relaxation of the Si-Si network strain but it reveals a derelaxation of the bond angle strain. Since the state of relaxation after annealing is very similar for pure and hydrogenated materials, our results give strong experimental support to the predicted configurational gap between a-Si and crystalline silicon. (C) 2010 American Institute of Physics. [doi:10.1063/1.3464961]
\end{abstract}

When amorphous silicon (a-Si) obtained by $\mathrm{Si}$ ion implantation in crystalline silicon (c-Si) is thermally annealed, it evolves spontaneously toward states of lower energy through a progressive diminution of the bond angle dispersion, $\Delta \theta$, around the tetrahedral angle of $\mathrm{c}-\mathrm{Si}$, and through point defect recombination. ${ }^{1-3}$ It is said that the covalent network after annealing is "more relaxed."

In fact, according to the theoretical models of a-Si, there is a "relaxed state" of a-Si characterized by a minimum value $\Delta \theta_{\text {relax }} \approx 6.6^{\circ}{ }^{4}$ Below $6.6^{\circ}$, no models can be built, i.e., a configurational gap is predicted between the allowed microscopic configurations of a-Si $\left(\Delta \theta>6.6^{\circ}\right)$ and c-Si $(\Delta \theta=0)$. This value has not been experimentally reached to date, and the question arises of whether this discrepancy is because the $\Delta \theta$ value of the relaxed state is higher than predicted, or it is due to kinetic reasons. Recently, we have shown that before crystallization begins, structural relaxation processes stop near $8.7^{\circ}$ because most of the mobile defects, whose diffusion facilitates network restructuring, have already recombined. ${ }^{3,5}$ This conclusion leaves the door open to the possibility of reaching a higher degree of relaxation if alternative mechanisms for network restructuring were to come into play. In that case, further diminution of $\Delta \theta$ would be possible and, eventually, a value of $\Delta \theta$ below $6.6^{\circ}$ could be reached if the real minimum value of $\Delta \theta$ were lower than predicted.

In contrast to pure a-Si, similar studies on hydrogenated amorphous $\mathrm{Si}(\mathrm{a}-\mathrm{Si}: \mathrm{H})$ are very scarce. It appears that the main structural transformations prior to crystallization are driven by dehydrogenation and atomic hydrogen diffusion. Apparently, densification up to the mass density of pure a-Si is indicative of long-range rearrangements of the Si-Si network after dehydrogenation. ${ }^{6}$ Probably, this extensive restructuring process, absent in pure a-Si, gives the materials further opportunities to reach the relaxed state and, consequently, allows us to address the fundamental problem of the

\footnotetext{
a) Author to whom correspondence should be addressed. Electronic mail: pere.roura@udg.es.
}

minimum value of $\Delta \theta$ mentioned above. The aim of this letter is to show that structural relaxation in a-Si:H is qualitatively different to that in a-Si both in terms of the structural transformations taking place and the microscopic mechanisms at work.

A wide variety of a-Si and a-Si:H materials were obtained by various deposition techniques: a-Si films on glass by electron beam evaporation (EBE); a-Si:H films on glass by hot wire chemical vapor deposition (HWCVD); a-Si:H films (label "TF") on glass; a-Si:H flakes (label "F") collected from the inner walls of the reactor chamber; and a-Si:H nanoparticles (npSi) by plasma enhanced CVD (PECVD) (Table I). The hydrogenated films were deposited in conditions leading to device-quality material and all the samples were undoped. The hydrogen content of the samples, $\mathrm{n}_{\mathrm{H}} / \mathrm{n}_{\mathrm{Si}}$, was determined by elementary analysis

TABLE I. Raman TO half-width $(\Gamma / 2)$, its increment during annealing of the samples, and the hydrogen content, $\mathrm{n}_{\mathrm{H}} / \mathrm{n}_{\mathrm{Si}}$.

\begin{tabular}{lccc}
\hline \hline \multicolumn{1}{c}{ Sample } & $\begin{array}{l}\Gamma / 2 \\
\left(\mathrm{~cm}^{-1}\right)\end{array}$ & $\begin{array}{l}\delta(\Gamma / 2) \\
\left(\mathrm{cm}^{-1}\right)\end{array}$ & $\mathrm{n}_{\mathrm{H}} / \mathrm{n}_{\mathrm{Si}}$ \\
\hline aSi(F) & a-Si:H grown by PECVD & \\
pmaSW(TF) & 34 & +0.5 & 0.12 \\
tfa(TF) & 34 & +1 & $\approx 0.15$ \\
pm(TF) & 34 & +0.8 & $\ldots$ \\
npSi & 33.4 & +2.2 & $\approx 0.15$ \\
& 34.5 & +1.5 & 0.28 \\
pSiHW & & & \\
aSiHW & a-Si:H grown by HWVD & $\ldots$ \\
& 34.7 & +1.0 & 0.13 \\
EBE350 & 34.0 & +2.5 & \\
EBE425 & & & \\
GRE350 & a-Si grown by EBE & 0 \\
GRE425 & 38.3 & -2.5 & 0 \\
GRE500 & 37.5 & -1.5 & 0 \\
\hline \hline
\end{tabular}




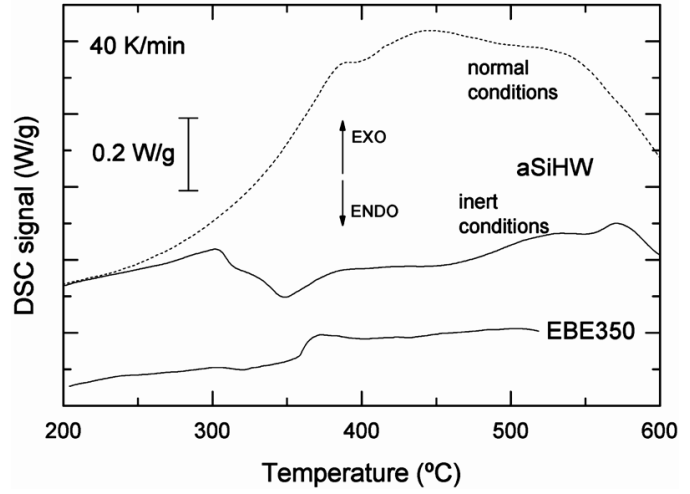

FIG. 1. Heat evolved of a pure a-Si sample (EBE350) and of a hydrogenated sample (aSiHW) measured in normal inert conditions and in a highly inert atmosphere to minimize residual oxidation (exo/endothermic contributions point up/downward).

(EA2400 Perkin Elmer). The annealing experiments were done by heating the samples in inert atmosphere at a constant rate (usually at $40 \mathrm{~K} / \mathrm{min}$ ) in a differential scanning calorimeter (DSC822 from Mettler Toledo) that delivers the heat exchanged as a continuous trace. Once the maximum temperature was reached, the sample was cooled down to room temperature without any isothermal stage. Finally, the material structure was analyzed by Raman spectroscopy (microRaman DILOR) at $632 \mathrm{~nm}$. Special care was taken to avoid local heating of the sample due to the laser beam. In particular, the anti-Stokes signal was recorded in selected samples to verify this condition.

Great attention has been paid to remove oxygen from the DSC furnace atmosphere because oxidation is enhanced during dehydrogenation of a-Si:H. ${ }^{7}$ For a "nominally" inert $\mathrm{N}_{2}$ atmosphere, we always obtained a high exothermic signal extending up to the crystallization temperature $\left(\approx 700{ }^{\circ} \mathrm{C}\right) .{ }^{8,9}$ This signal disappeared when more inert conditions were used (higher $\mathrm{N}_{2}$ flow rates) revealing a less intense signal containing endothermic contributions (Fig. 1).

In Fig. 1 we plot the DSC thermograms of two samples showing the heat evolved from the material before crystallization begins, $\mathrm{Q}_{\text {relax }}$. A small exothermic signal beginning at around $360{ }^{\circ} \mathrm{C}$ and extending above $500{ }^{\circ} \mathrm{C}$ has been measured for the a-Si sample. Notice that, as expected, the signal threshold is close to the substrate deposition temperature $\left(350{ }^{\circ} \mathrm{C}\right)$ used to deposit this particular sample. The area below the curve extrapolated up to $600{ }^{\circ} \mathrm{C}$ delivers a relaxation heat of $\mathrm{Q}_{\text {relax }}=40 \pm 20 \mathrm{~J} / \mathrm{g}$. In contrast, the DSC signal of the hydrogenated sample has a more complex structure. Although it is very difficult to know with accuracy the absolute zero of the DSC signal, its comparison with the curve affected by residual oxidation makes clear that an endothermic contribution begins around $300{ }^{\circ} \mathrm{C}$.

To analyze the changes in the $\mathrm{Si}-\mathrm{Si}$ covalent network caused by annealing, we measured the Raman spectra of the samples in their as-grown state and after the heat treatment. Typical spectra are shown in Fig. 2. After annealing, the half-width, $\Gamma / 2$, of the pure a-Si samples remains unchanged or diminishes, whereas it increases for all the hydrogenated samples (Table I). The results are collected in Fig. 3 together with the evolution observed for a-Si films obtained by ion implantation. ${ }^{1}$ We have confidence in the ordering of the values obtained for our samples, which has been verified by superposing the spectra as in Fig. 2. However, we consider

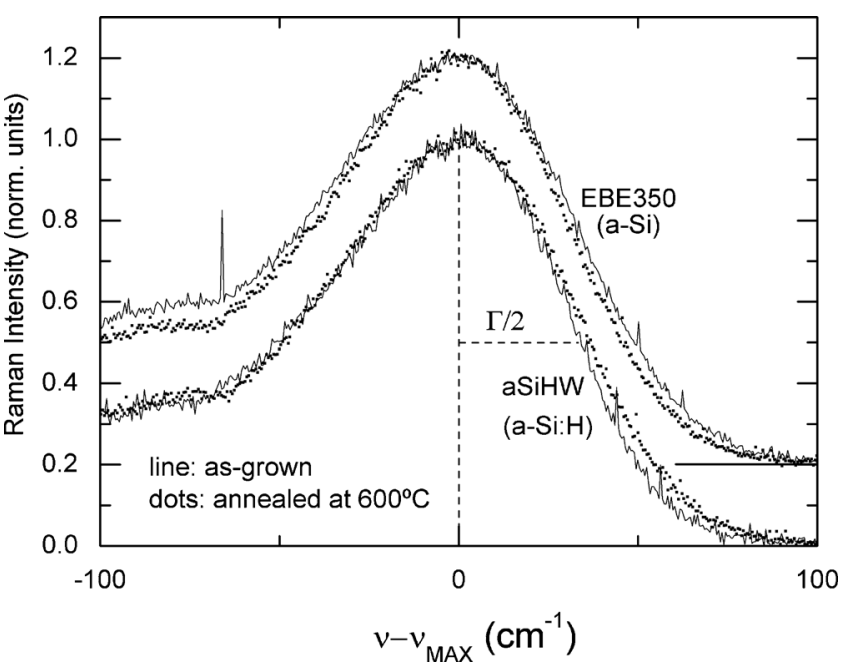

FIG. 2. The width of the Raman TO band increases (decreases) during annealing for the a-Si:H (a-Si) samples.

that the values for ion-implanted a-Si in relation to our samples may contain an uncertainty of $\pm 1 \mathrm{~cm}^{-1}$. In addition, according to our analysis published in Ref. 5 the temperature of these samples has been increased by $65^{\circ} \mathrm{C}$ to the published values ${ }^{1}$ to take into account that anneals lasted $45 \mathrm{~min}$.

In accordance with the widely accepted interpretation of the Raman TO band width, the values of $\Gamma / 2$ have been converted to $\Delta \theta$ (right $y$-axis of Fig. 3) by using the following particular relationship of Beeman et al.: ${ }^{4}$

$$
\Gamma / 2\left(\text { in } \mathrm{cm}^{-1}\right)=7.5+3 \Delta \theta(\text { in } \mathrm{deg}) \text {. }
$$

It is clear from Fig. 3 that $\Delta \theta$ decreases with annealing for pure a-Si whereas it increases for the hydrogenated materials. In this context it is worth to comment that recent calculations ${ }^{10}$ have analyzed at the microscopic level the effect of hydrogenation on the network strain. It is shown that hydrogen incorporation near small voids and divacancies tends to occur at the most strained bonds, thus reducing strain. It is thus expected that the strain $(\Delta \theta)$ would increase after dehydrogenation.

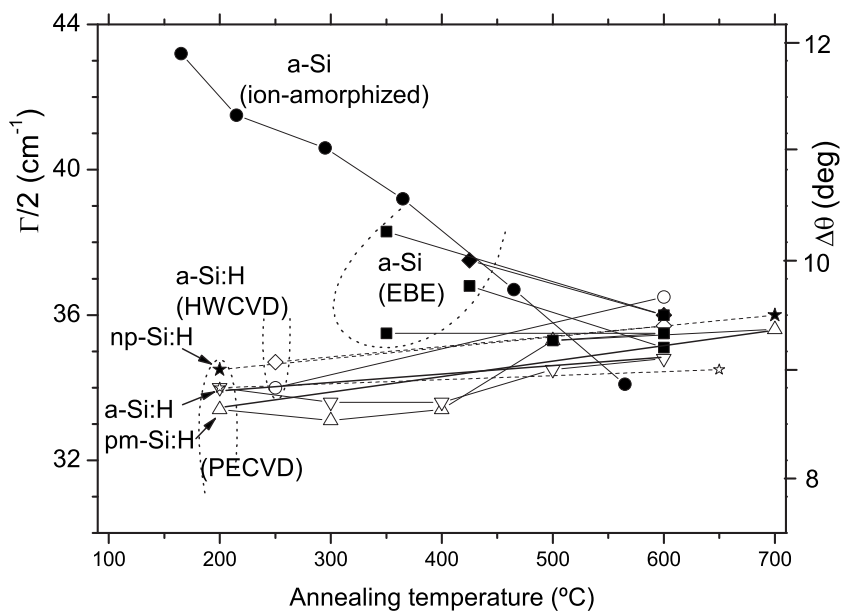

FIG. 3. Evolution of the TO half-width during annealing and the corresponding dispersion of $\mathrm{Si}-\mathrm{Si}$ bond-angles according to the Beeman formula [Eq. (1)]. For each sample, the lowest temperature is the deposition temperature, i.e., the point corresponds to the as-grown condition. The ionamorphized a-Si points are taken from Ref. 1 . 
Given that $\Delta \theta$ diminishes for a- $\mathrm{Si}$, the interpretation of $\mathrm{Q}_{\text {relax }}$ in these samples is straightforward. The bond-angle dispersion entails a built-in strain and, consequently, a strain energy that can be quantified as follows: ${ }^{11}$

$$
U_{\Delta \theta}=A(\Delta \theta)^{2},
$$

where $A$ is a constant that depends on the $\mathrm{Si}-\mathrm{Si}$ bond-force constants. We have recently shown ${ }^{3}$ that the diminution of $U_{\Delta \theta}$ is the main contribution to the relaxation heat of ionimplanted a-Si and we obtained $A \approx 3.0 \mathrm{~J} /\left(\mathrm{g} \mathrm{deg}^{2}\right)$. If we apply this value to the EBE350 sample, for which $|\delta \Delta \theta|$ $\approx 0.8^{\circ}$, a value around $25 \mathrm{~J} / \mathrm{g}$ is predicted for $\mathrm{Q}_{\text {relax }}$, which agrees with the area below the DSC curve in Fig. 1 $(40 \pm 20 \mathrm{~J} / \mathrm{g})$.

In the case of hydrogenated samples, in addition to small residual oxidation, several following processes may contribute to $\mathrm{Q}_{\text {relax }}$ : (a) the increment of $\Delta \theta$, (b) the increment of dangling-bond (DB) density, and (c) dehydrogenation. The first two processes are endothermic and weak. According to Table I and Eq. (2), the contribution of $\Delta \theta$ would be lower than $30 \mathrm{~J} / \mathrm{g}$ for all the samples. On the other hand, we know that the DB density increases up to $5 \times 10^{19} \mathrm{~cm}^{-3}$ upon annealing at high temperature, ${ }^{12}$ and this results in a small contribution of $4 \mathrm{~J} / \mathrm{g}$ to the relaxation heat [formation energy of one $\mathrm{DB} \approx 1 \mathrm{eV}$ (Ref. 8)]. Finally, the small exothermic values reported for dehydrogenation $[\approx 50 \mathrm{meV} / \mathrm{H}$-atom (Ref. 13)] would lead to contributions of about $20 \mathrm{~J} / \mathrm{g}\left(\mathrm{n}_{\mathrm{H}} / \mathrm{n}_{\mathrm{Si}}\right.$ $\approx 10 \%$ ). This analysis explains why (a) the DSC curve is more complex than that of the pure a-Si samples, (b) the DSC signal is very weak, and (c) weak endothermic contributions are expected.

The increment of $\Delta \theta$ in a-Si:H deduced from the Raman spectra is consistent with the increment of the weak bond (WB) density reported by other authors. ${ }^{12,14}$ In fact, WBs are highly strained $\mathrm{Si}-\mathrm{Si}$ bonds and, consequently, they correspond to the tail of the $\theta$ distribution. When films are annealed in vacuum (or in inert atmosphere), most hydrogen is lost and the density of WBs (measured through the Urbach tail slope) increases. ${ }^{12}$ However, when the experiment is done in hydrogen plasma, which prevents intense dehydrogenation, the WB density remains unchanged. ${ }^{14}$ So, restructuring of the $\mathrm{Si}-\mathrm{Si}$ network in a-Si:H is not directly related to the network temperature but to dehydrogenation. The contrary holds for a-Si, whose structural relaxation is intimately related to the diffusion of network defects and, consequently, on temperature. ${ }^{5}$

We have arrived, finally, at the interesting question posed in the introduction about the significance of the $\Delta \theta$ value obtained for the relaxed samples. First, it should be noted that, after annealing, we obtain $\Delta \theta$ values in the narrow range between $9.0^{\circ}$ and $9.7^{\circ}$, irrespective of the deposition technique. Second, since few hydrogen atoms are left in the a-Si:H samples after annealing, it is tempting to interpret this value as the minimum value for any allowed configuration of pure a-Si. The fact that this state is achieved with the hydrogenated samples supports this assertion because after dehydrogenation (a) $99.9 \%$ of the DBs left behind by the hydrogen atoms recombine and (b) the material's mass density increases up to the value of pure $\mathrm{a}-\mathrm{Si}^{6}{ }^{6}$ This means that during annealing the $\mathrm{Si}-\mathrm{Si}$ network undergoes extensive re- structuring, thus allowing the material to explore all possible microscopic configurations. Consequently, if a-Si configurations of lower $\Delta \theta$ existed, they would be reached during network restructuring because of their lower energy. And finally, an independent experiment that demonstrates the high stability of the configuration with $\Delta \theta \approx 9^{\circ}-9.7^{\circ}$ has been done with the a-Si EBE samples. Isothermal anneals at $600{ }^{\circ} \mathrm{C}$ lasting as long as $50 \mathrm{~h}$ have not been able to reach lower values of $\Delta \theta$. Consequently, we think that our results provide strong experimental evidence for the existence of a configuration gap between $\mathrm{c}-\mathrm{Si}$ and $\mathrm{a}-\mathrm{Si}$ defined by $\Delta \theta \approx 9 \circ .{ }^{15}$ Furthermore, since $\Delta \theta$ increases after dehydrogenation for the hydrogenated samples, our results show that, as expected, the presence of $\mathrm{Si}-\mathrm{H}$ groups in the covalent network reduce the bond-angle strain and even reduce the configurational gap.

In summary, thermal annealing experiments on a-Si reveal a decrease in $\mathrm{Si}-\mathrm{Si}$ network strain whereas an increase is observable in a-Si:H. In other words, the $\mathrm{Si}-\mathrm{Si}$ network undergoes relaxation in a-Si but it "derelax" in a-Si:H. Our experiments also provide strong support for the existence of the predicted configurational gap between $\mathrm{a}-\mathrm{Si}(\mathrm{H})$ and $\mathrm{c}-\mathrm{Si}$. This gap would decrease for hydrogenated material.

Note added. During the revision of the manuscript, we have known that the WB density in a-Si:H films can be highly reduced by ion-bombardment during growth. ${ }^{16} \mathrm{We}$ wonder if $\Delta \theta$ in samples prepared in this way would be significantly lower than our reported values.

We acknowledge the financial support from MICINN (Grant No. MAT2006-11144), MEC (Grant No. SB20060062), and the Generalitat de Catalunya (Grant No. 2005SGR-00666).

${ }^{1}$ S. Roorda, W. C. Sinke, J. M. Poate, D. C. Jacobson, S. Dierker, B. S. Dennis, D. J. Eaglesham, F. Spaepen, and P. Fuoss, Phys. Rev. B 44, 3702 (1991)

${ }^{2}$ P. A. Stolk, F. W. Saris, A. J. M. Berntsen, W. F. van der Weg, L. T. Sealy, R. C. Barklie, G. Krötz, and G. Müller, J. Appl. Phys. 75, 7266 (1994).

${ }^{3}$ P. Roura, J. Farjas, and P. Roca i Cabarrocas, J. Appl. Phys. 104, 073521 (2008).

${ }^{4}$ D. Beeman, R. Tsu, and M. F. Thorpe, Phys. Rev. B 32, 874 (1985).

${ }^{5}$ P. Roura and J. Farjas, Acta Mater. 57, 2098 (2009).

${ }^{6}$ Z. Remes, M. Vanecek, A. H. Mahan, and R. S. Crandall, Phys. Rev. B 56, R12710 (1997)

${ }^{7}$ D. Das, J. Farjas, P. Roura, G. Viera, and E. Bertran, Appl. Phys. Lett. 79, 3705 (2001)

${ }^{8}$ P. Roura, J. Farjas, C. Rath, J. Serra-Miralles, E. Bertran, and P. Roca i Cabarrocas, Phys. Rev. B 73, 085203 (2006).

${ }^{9}$ P. Roura, J. Farjas, and P. Roca i Cabarrocas, Thin Solid Films 517, 6239 (2009).

${ }^{10}$ S. Chakraborty and D. A. Drabold, Phys. Rev. B 79, 115214 (2009).

${ }^{11}$ T. Saito, T. Karasawa, and I. Ohdomari, J. Non-Cryst. Solids 50, 271 (1982)

${ }^{12}$ K. Zellama, L. Chahed, P. Sladek, M. L. Theye, J. H. von Bardeleben, and P. Roca i Cabarrocas, Phys. Rev. B 53, 3804 (1996).

${ }^{13}$ J. Farjas, D. Das, J. Fort, P. Roura, and E. Bertran, Phys. Rev. B 65, 115403 (2002).

${ }^{14}$ N. H. Nickel and W. B. Jackson, Phys. Rev. B 51, 4872 (1995).

${ }^{15}$ Although this value is clearly higher than the theoretical $6.6^{\circ}$, the precise discrepancy depends on the precision of the $\Delta \theta(\Gamma / 2)$ relationship used here [Eq. (1)].

${ }^{16}$ G. Ganguly, I. Sakata, and A. Matsuda, J. Non-Cryst. Solids 198-200, 300 (1996). 